\title{
Discours
}

Revue de linguistique, psycholinguistique et informatique. A journal of linguistics, psycholinguistics and computational linguistics

$6 \mid 2010$

Le fonctionnement en discours des énoncés averbaux autonomes

\section{Le fonctionnement en discours des unités prédicatives averbales autonomes}

Présentation du numéro

\section{Eva Havu et Florence Lefeuvre}

\section{(2) OpenEdition}

Journals

Édition électronique

URL : http://journals.openedition.org/discours/7716

DOI : $10.4000 /$ discours. 7716

ISSN : 1963-1723

Éditeur :

Laboratoire LATTICE, Presses universitaires de Caen

Référence électronique

Eva Havu et Florence Lefeuvre, «Le fonctionnement en discours des unités prédicatives averbales autonomes », Discours [En ligne], 6 | 2010, mis en ligne le 29 septembre 2010, consulté le 19 avril 2019. URL : http://journals.openedition.org/discours/7716 ; DOI : 10.4000/discours.7716

Ce document a été généré automatiquement le 19 avril 2019

\section{cc) (†)}

Discours est mis à disposition selon les termes de la licence Creative Commons Attribution - Pas d'Utilisation Commerciale - Pas de Modification 4.0 International. 


\section{Le fonctionnement en discours des unités prédicatives averbales autonomes}

Présentation du numéro

Eva Havu et Florence Lefeuvre

1 La question de la phrase averbale a donné lieu à une large production scientifique essentiellement axée sur les langues où les phrases attributives sans verbe être sont des constructions standard, comme l'arabe, l'hébreu, le hongrois, le russe, etc. (cf.par exemple, Eid, 1991 ; Hengeveld, 1992 ; Nordlinger et Sadler, 2006). Dans les langues où les phrases attributives avec le verbe être sont des constructions standard, telles que l'allemand, l'anglais, le français, l'italien, etc., cette question est parfois traitée de façon partielle ou ramenée à des schémas basés sur l'ellipse (cf. Barton, 1990 ; Greenbaum et al. ,1985 ; Merchant, 2004). Récemment néanmoins, ont émergé des approches typologiques qui distinguent les prédications averbales des constructions purement elliptiques (Behr et Quintin, 1996 pour l'allemand ; Cresti, 1998 pour l'italien ; Lefeuvre, 1999 pour le français ; Delorme, 2004 pour l'anglais), et plusieurs points d'ordre syntaxique ou sémantique ont déjà fait l'objet d'une attention particulière (Behr et Lefeuvre, à paraître; Behr et Lefeuvre, 2005 ; Behr et al., 2004 ; Lefeuvre (ed.), 2004).

2 Ce numéro thématique a pour objectif d'apporter des éléments nouveaux sur le fonctionnement en discours des unités prédicatives averbales autonomes. Cette problématique rejoint en partie celle du découpage des unités minimales en discours (cf. Degand et Simon, 2005). Plus précisément, sont abordés dans ce numéro deux grands types de questions liées aux prédications averbales autonomes: leur statut par rapport aux prédications secondes ou par rapport à des énoncés verbaux, ainsi que leurs fonctions discursives et pragmatiques. Deux travaux traitent du français, l'un du français littéraire, l'autre du français oral. Trois articles examinent une langue étrangère : le corpus japonais se constitue de slogans, le corpus finnois de traductions de romans et le corpus japonais de textes journalistiques. Les cinq contributions se fondent donc sur des types de textes différents. 
3 B. Combettes et A. Kuyumcuyan examinent dans leur article les unités prédicatives averbales d'apparence autonome dans les récits des enquêtes du commissaire Maigret rédigées par G. Simenon entre 1930 et 1972. Ils cherchent à clarifier le statut de ces unités en se demandant s'il s'agit de phrases averbales ou d'appositions « retardées »? Leur étude met en lumière le rôle déterminant du contexte par rapport aux considérations formelles, ce qui amène à privilégier la notion d'un continuum allant de la phrase nominale à l'apposition, de la prédication averbale « première » à la structure dite de « prédication seconde ».

4 E. Havu s'intéresse également à la frontière entre prédications averbales « premières » et "prédications secondes ", en prenant comme point de départ le finnois, où le marquage casuel distingue en principe les structures ayant un point d'ancrage dans le contexte de gauche des structures entièrement autonomes. Toutefois, les traductions finnoises de trois romans français montrent que les traducteurs n'ont guère osé réfléchir sur l'autonomie ou la non-autonomie des structures détachées par un point ou une virgule: dans la traduction des premières, on ne trouve pratiquement que le cas sujet, tandis que dans la traduction des prédications secondes, la dépendance d'un élément dans le contexte de gauche est marquée d'une manière explicite.

5 A. Herdam traite des slogans électoraux allemands en usage entre 1919 et nos jours. Ces textes, où il est important de présenter des informations complexes d'une manière concise, tout en tenant compte de l'impact visuel et d'aspects prosodiques, sont particulièrement riches en énoncés averbaux. Son approche diachronique et pragmatique montre que les énoncés averbaux, qui peuvent accomplir pratiquement tous les actes de communication, deviennent particulièrement fréquents à partir des années 70. Ils présentent des avantages par rapport aux énoncés verbaux, telle que l'autorisation d'une nouvelle liberté à l'égard du choix tutoiement / vouvoiement, et à l'égard des contraintes d'une argumentation conventionnelle.

6 M. Kaneko étudie les diverses fonctions discursives remplies en japonais par une phrase averbale du type «proposition subordonnée + syntagme nominal». Les travaux antérieurs ont mis cette construction en parallèle avec une phrase averbale du type « Le lavabo qui déborde! »en français. En se fondant sur un corpus journalistique et en profitant des analyses antérieures sur la phrase averbale dans d'autres langues, l'auteur soutient qu'il faut distinguer, à côté d'un premier type déictique, trois autres types: i) celui qui présente une situation comme formant un bloc sémantique et qui introduit un nouveau plan énonciatif, mais n'effectue pas l'ancrage dans le moment de la perception; ii) celui qui introduit un plan énonciatif déplacé, mais ne met pas en relief la situation dénotée, et fait du référent du syntagme nominal un topique discursif ou un cadre spatiotemporel ; iii) celui qui ne présente aucune caractéristique du premier type et se trouve réservé à la mise en relief d'un topique discursif portant sur le texte entier.

7 N. Tanguy rapproche deux types de structures couramment employées en français parlé et qui présentent la particularité d'être énoncées en deux temps. L'une est construite avec un verbe (à la caisse ils se pèsent) et l'autre est averbale (très bien ta vie). L'auteure montre que ces deux constructions, qui présentent les mêmes rendements communicatifs, suivent un même schéma prosodique comportant deux groupes intonatifs. Même si la première est porteuse d'une intonation modale qui la rend autonome, et la seconde est prononcée comme un appendice, elles résultent d'une même opération de focalisation visant à placer en tête de phrase un argument à vocation rhématique. 
Ce numéro thématique illustre donc quelques problématiques liées aux énoncés averbaux autonomes et montre que ces énoncés sont très présents dans différents types de texte et même dans des langues d'origine différente. Il ébauche des pistes de recherches qui mériteraient bien une étude plus approfondie.

\section{BIBLIOGRAPHIE}

BARTON, E. 1990. Nonsentential Constituents. Amsterdam / Philadelphia : John Benjamins.

BEHR, I., QUINTIN, H. 1996. Verblose Sätze im Deutschen. Tübingen : Stauffenburg.

BEHR, I., FRANÇOIS, J., LACHERET, A., LEFEUVRE, F. 2004. Introduction. Syntaxe et Sémantique : Aux

Marges de la prédication, Syntaxe et Sémantique $6: 7-12$.

BEHR, I., LEFEUVRE, F. 2005. La configuration GN GN : comment reconnaître le prédicat ? Les Constituants prédicatifs et la diversité des langues. In I. BEHR, A. FRANÇOIS (eds), Mémoires de la Société linguistique de Paris, tome XIV. Louvain : Peeters : 199-221.

BEHR, I., LEFEUVRE, F. à paraître. Les énoncés averbaux autonomes entre grammaire et discours. Paris : Ophrys.

COMBETTES, B. 2007. Les ajouts après le point : aspects syntaxiques et textuels. In M. CHAROLLES, N. FOURNIER, C. FUCHS, F. LEFEUVRE (eds), Parcours de la phrase. Mélanges offerts à Pierre Le Goffic. Paris : Ophrys.

CRESTI, E. 1998. Gli enunciati nominali. In M.T. NAVARRo (ed.), Atti del IV Convegno internazionale SILFI (Madrid 27-29 giugno 1996). Pisa : Cesati : 171-191.

DEGAND, L., SIMON, A.C. 2005. Minimal Discourse Units : Can we define them, and why should we ? In M. AURNAGUE., M. BRAS, A. LE DRAOULEC, L. VIEU (eds), Connectors, discourse framing and discourse structure : from corpus-based and experimental analyses to discourse theories, Proceedings of SEM-05, Biarritz, 14-15 novembre 2005 : 65-74.

DELORME, B. 2004. Les énoncés nominaux dans la fiction contemporaine de langue anglaise, implications sémantiques et pragmatiques de la prédication averbale. Thèse de doctorat, Paris 4-Sorbonne.

EID, M. 1991. Verbless Sentences in Arabic and Hebrew. In B. COMRIE, M. EID (eds), Perspectives on Arabic Linguistics III. Papers from the Third Symposium on Arabic Linguistics.

Amsterdam / Philadelphia : John Benjamins : 31-61.

GREENBAUM, S., QUIRK, R., LEECH, G. et SVARTVIK, J. 1985. A Comprehensive Grammar of the English language. London : Longman.

HAVU, E., PIERRARD, M. 2007. Prédication seconde et type de discours : les adjoints participiaux dans les médias oraux. In M. BROTH, M. FORSGREN, C. NORÉN, F. SULLET-NYLANDER (eds), Le français parlé des médias, Actes du colloque de Stockholm, 8-12 juin 2005. Stockholm : Almqvist \& Wiksell International : 273-288.

HENGEVELD, K. 1992. Non-verbal predication : theory, typology, diachrony. Berlin : Mouton de Gruyter. LEFEUVRE, F. 1999. La Phrase averbale en français. Paris : L'Harmattan. 
LEFEUVRE, F. (ed.) 2004. Préface. Verbum : La phrase averbale : délimitations et caractéristiques XXVI (4) : 265-268.

LEFEUVRE, F. 2007. Le segment averbal comme unité syntaxique textuelle. In M. CHAROLLES, N. FOURNIER, C. FUCHS, F. LEFEUVRE (eds), Parcours de la phrase. Mélanges offerts à Pierre Le Goffic. Paris : Ophrys : 143-158.

MERCHANT, J. 2004. Fragments and Ellipsis. Linguistics and Philosophy 27 (6) : 661-738.

NORDLINGER, R., SADLER, L. 2006. Verbless Clauses : revealing the structure within. Accessible en ligne sur http://privatewww.essex.ac.uk/ louisa/newpapers/nordlinger-sadler-final.pdf.

\section{AUTEURS}

\section{EVA HAVU}

Université de Helsinki - Paris 3 / UMR CNRS Lattice 8094 - Gramm-R

\section{FLORENCE LEFEUVRE}

Université Paris 3 / CLESTHIA - Gramm-R 